\title{
Influence of gaseous annealing environment on the properties of indium-tin-oxide thin films
}

\author{
R. X. Wang, C. D. Beling, S. Fung, ${ }^{\text {a) }}$ A. B. Djurišić, C. C. Ling, and S. Li \\ Department of Physics, The University of Hong Kong, Pokfulam Road, Hong Kong, \\ People's Republic of China
}

(Received 7 July 2004; accepted 25 October 2004; published online 30 December 2004)

\begin{abstract}
The influence of postannealing in different gaseous environments on the optical properties of indiu-tin-oxide (ITO) thin films deposited on glass substrates using e-beam evaporation has been systematically investigated. It is found that the annealing conditions affect the optical and electrical properties of the films. Atomic force microscopy, x-ray diffraction, and x-ray photoemission spectroscopy (XPS) were employed to obtain information on the chemical state and crystallization of the films. These data suggest that the chemical states and surface morphology of the ITO film are strongly influenced by the gaseous environment during the annealing process. The XPS data indicate that the observed variations in the optical transmittance can be explained by oxygen incorporation into the film, decomposition of the indium oxide phases, as well as the removal of metallic In. (C) 2005 American Institute of Physics. [DOI: 10.1063/1.1834984]
\end{abstract}

\section{INTRODUCTION}

As a result of the rapid development of optoelectronic devices, ${ }^{1-3}$ such as solar cells, ${ }^{3}$ liquid-crystal display devices, organic and inorganic light-emitting devices, ${ }^{2}$ laser diodes, and ultraviolet photodetectors, indium-tin-oxide (ITO) thin film is attracting increasing attention ${ }^{1-5}$ as a low resistance contact material with high transparency in the visible spectral region. Furthermore, ITO has many unique properties such as excellent substrate adhesion, thermal stability and ease of patterning. Several methods such as thermal evaporation, ${ }^{4,6} \mathrm{rf}$ and $\mathrm{dc}$ sputtering, ${ }^{2,7,8}$ e-beam evaporation, ${ }^{1,3,9-12}$ and so forth have been employed to deposit ITO films on different substrates. Each method has its advantages and disadvantages. Available studies show that preparation methods and conditions strongly affect the microstructures and chemical compositions of deposited ITO films and thus significantly modify the electrical and optical characteristics of the films. ${ }^{4,6-11}$ To obtain optimal electrical and optical properties of an ITO film, the growth parameters and conditions must also be studied. There have been some reports ${ }^{7,8,12}$ discussing the effects of preparation conditions and postdeposition annealing in oxygen atmosphere on the optical and electrical properties of ITO films. The electrical properties of deposited ITO films are strongly influenced by the density of charge carriers, i.e., tin dopants and oxygen vacancy donors. ${ }^{7}$ For example, Mori et al. ${ }^{12}$ reported that oxygen incorporation during the film annealing plays an important role in the optical properties of the ITO films annealed in $\mathrm{O}_{3}, \mathrm{O}_{2}$, and air. Huang et al. ${ }^{8}$ reported on the influence of oxygen flux rate during sputtering deposition on surface morphology and grain size.

Sputtering preparation method is studied by most research groups due to its many advantages, such as faster deposition rate. However, it may significantly damage the

\footnotetext{
${ }^{a)}$ Author to whom correspondence should be addressed; electronic mail: sfung@hkucc.hku.hk
}

substrate surface ${ }^{13}$ and cause defects ${ }^{14}$ which will influence the performance of optoelectronic devices. E-beam evaporation is often preferred in preparing ITO films for application in III-V compound devices. ${ }^{1}$ During reactive e-beam evaporation, oxygen gas is usually guided into the chamber as a reactive gas that promotes the growth of high-quality ITO film. This procedure, however, can induce other unexpected problems because oxygen easily reacts with many elements under high temperature. For this reason, in the present work, a study is made on the influence of different annealing gases on the transmittance and electrical properties of e-beamevaporated ITO films for which no oxygen overpressure has been used during growth. It is found that annealing asdeposited ITO films in different gaseous environments at $200{ }^{\circ} \mathrm{C}$ can significantly influence their optical and electrical properties. In order to understand these variations, detailed studies on ITO microstructure were carried out by atomic force microscopy (AFM), x-ray diffraction (XRD), and x-ray photoemission spectroscopy (XPS).

\section{EXPERIMENTAL DETAILS}

$\mathrm{In}_{2} \mathrm{O}_{3}: \mathrm{SnO}_{2}(9: 1)$ powder was used as the evaporation source. Before being loaded into the chamber, the glass substrate was cleaned in an ultrasonic cleaner for $10 \mathrm{~min}$ with acetone, ethanol, and deionized (DI) water and then dried with nitrogen gas. The background vacuum in the chamber was $2 \times 10^{-5}$ Torr. The substrates were not intentionally heated during the e-beam evaporation. The ITO film thickness was controlled to be $400 \AA$ using a quartz-crystal monitor. After deposition, the films were annealed in a furnace at $200{ }^{\circ} \mathrm{C}$ for $30 \mathrm{~min}$ under the flow of different gases. The samples prepared for comparison of annealing in different atmospheres were deposited in the same deposition process. The annealing gases studied were air, $\mathrm{Ar}, \mathrm{N}_{2}$, and $\mathrm{N}_{2}: \mathrm{H}_{2}$ (forming gas).

The crystalline structures of the films were examined with a Philips PW1825 x-ray diffractometer at room tem- 


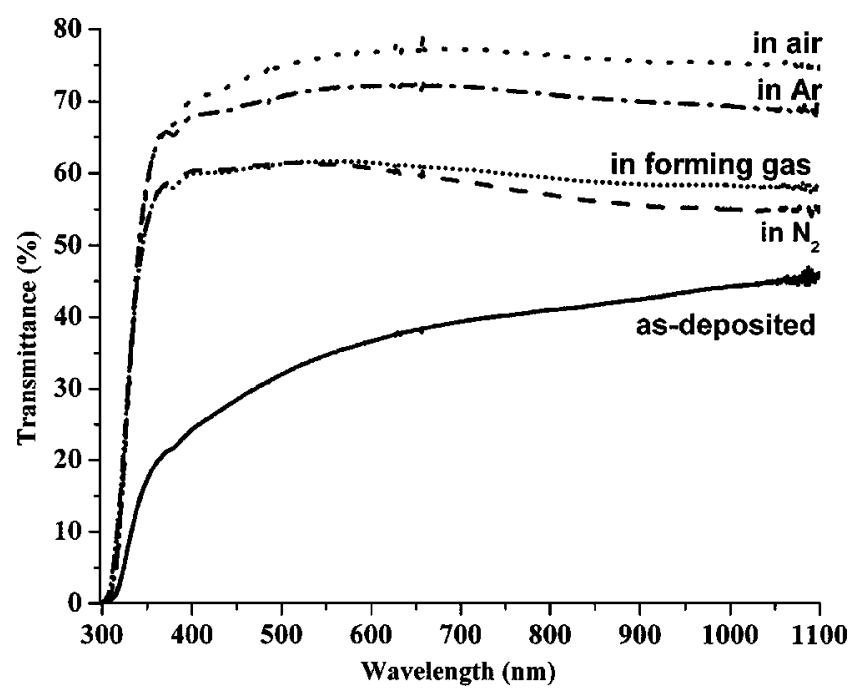

FIG. 1. Transmittance of ITO films as-deposited and annealed in different gas environments.

perature. The chemical compound information for oxygen, indium, and tin was studied using a PHI 5600 XPS with monochromatic $\mathrm{Al} K \alpha$ x-ray source for superior energy resolution. The typical operation parameters were $350 \mathrm{~W}$ for the $\mathrm{X}$-ray source and the tilted angle of $45^{\circ}$ for the analyzer. All XPS experiments were carried out under ultrahigh vacuum conditions at room temperature. The decomposition of the component peaks in the XPS spectra was carried out with a mixture of Gaussian and Lorentzian functions. The relative element ratio was calculated from the ratio of integrated areas of the corresponding component peaks and corrected with an instrument response factor. The transmittance of the ITO films in the wavelength range 190-1100 nm was measured in a HP 8453 UV-visible spectrophotometer system, without bare substrate as a reference. The electrical properties were probed using a 0.5-T magnetic-field BIO-RAD HL5580 Hall-effect system at room temperature. The surface morphology of the samples was observed with Digital Instrument Nanoscope III AFM. The images were obtained in tapping mode under ambient environment at room temperature with a scanning area of $5 \times 5 \mu \mathrm{m}^{2}$.

\section{RESULTS AND DISSCUSSION}

Figure 1 shows the transmittance curves of the asdeposited film and films annealed in different gases. It can be seen that the transmittance of all the annealed films is greatly improved compared to the as-deposited film. Moreover, unlike the case of the as-deposited film, whose transmittance strongly increases with wavelength in the 380-1100-nm range, the transmittance of the annealed films is only weakly dependent on wavelength. Also, the transmittance edge of the annealed films shifts to shorter wavelengths. This is the Burstein-Moss shift ${ }^{15}$ caused by the increased carrier concentration and the Fermi-level shift to higher energy in a more heavily doped $n$-type semiconductor. This observation is consistent with the electrical properties of the films as listed in Table I, where the carrier concentration and the mobility of the annealed films become significantly higher than those of the as-deposited film.
TABLE I. Properties of the ITO films deposited at $3.3 \AA$ As: as-deposited, annealed at $200{ }^{\circ} \mathrm{C}$ in $\mathrm{N}_{2}$, forming gas, Ar, and air.

\begin{tabular}{cccccc}
\hline \hline & As-deposited & $\mathrm{N}_{2}$ & Forming gas & Ar & Air \\
\hline Transmittance $(500 \mathrm{~nm})$ & $32 \%$ & $61 \%$ & $61 \%$ & $70 \%$ & $74 \%$ \\
$\rho\left(\times 10^{-4} \Omega \mathrm{cm}\right)$ & 555.4 & 4.9 & 5.8 & 7.6 & 9.3 \\
$\mu\left(\mathrm{cm}^{2} / \mathrm{V} \mathrm{s}\right)$ & 0.3 & 11.4 & 8.9 & 3.5 & 4.7 \\
$n\left(\times 10^{21} \mathrm{~cm}^{-3}\right)$ & 0.3 & 1.1 & 1.1 & 2.2 & 1.4 \\
$\mathrm{rms} \mathrm{roughness}(\AA)$ & 14 & 11 & 28 & 11 & 23 \\
$\mathrm{O} / \mathrm{In}+\mathrm{Sn}$ & 2.1 & 1.6 & 1.5 & 2.4 & 2.3 \\
$\mathrm{O}_{530.3} / \mathrm{O}_{530.8}$ & 3.5 & 0.9 & 1.3 & 1.6 & 0.6 \\
\hline \hline
\end{tabular}

Figure 1 also shows that the transmittance of annealed samples depends on the annealing gas. The transmittance of ITO film annealed in air and Ar gas is higher than that of the films annealed in $\mathrm{N}_{2}$ and forming gas. There are many factors, such as the microstructure difference, chemical composition ratio, and surface roughness that can account for the observed differences in the measured transmittance and electrical properties between the as-deposited film and annealed films. The surface morphology of the film annealed in different gaseous atmospheres was measured by AFM as shown in Fig. 2. From Table I and Fig. 2, it can be seen that the transmittance of the film annealed in air is the highest although its surface is rougher than that of the film annealed in Ar. It suggests that the surface roughness of the films seems not to have a close relationship with the transmittance of films in the present case. The influence of other factors on the transmittance of annealed films will be discussed in the following paragraphs.

To study the microstructure of the films, XRD measurements were performed. Figure 3 shows the XRD curves of the as-deposited and annealed ITO films. Despite the small thickness of the films the diffraction peaks from the (222) crystalline planes are still resolvable for the annealed samples. No diffraction peak except a broad background (centered on $2 \theta=25^{\circ}$ ) is observed for the as-deposited film
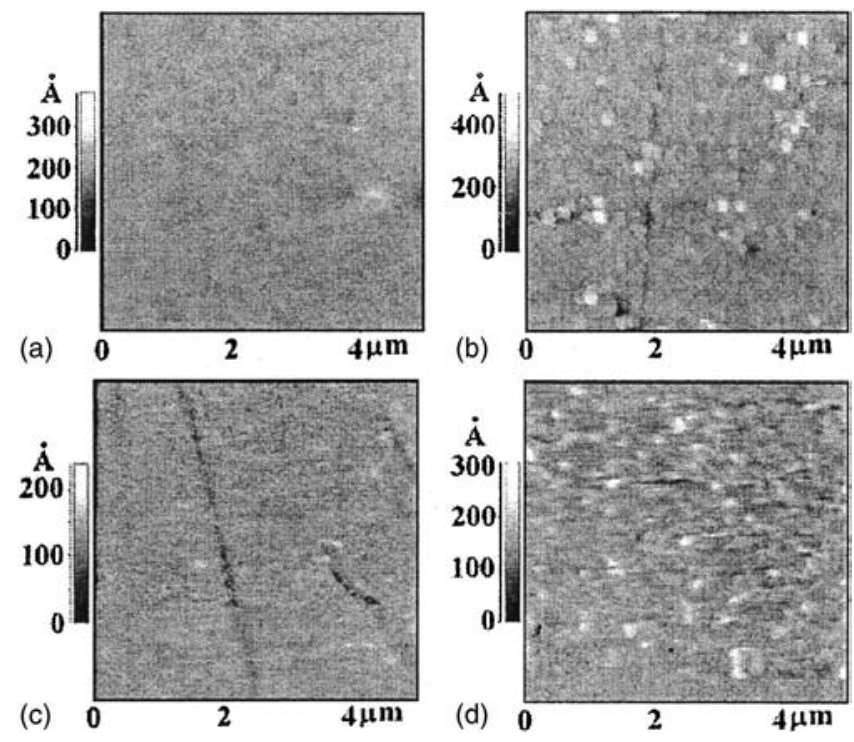

FIG. 2. AFM images of the films annealed in different gaseous environments at $200{ }^{\circ} \mathrm{C}$ (a) annealing in $\mathrm{N}_{2}$, (b) annealing in forming gas, (c) annealing in $\mathrm{Ar}$, and (d) annealing in air. 


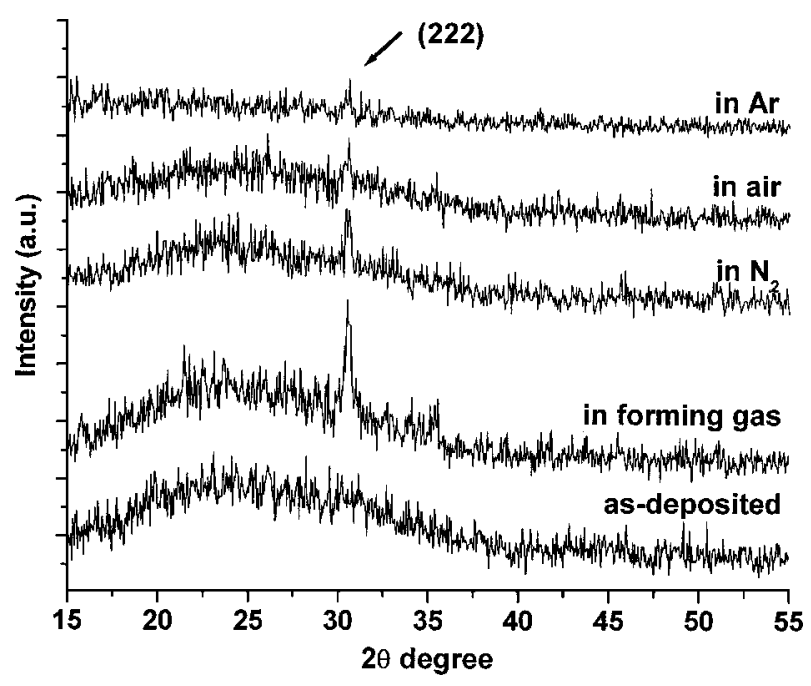

FIG. 3. XRD spectra of the ITO films as-deposited and annealed in different gas environments.

indicating a nanocrystalline ${ }^{11}$ and/or an amorphous ${ }^{4}$ structure for the as-deposited ITO film. It is known that the (222) orientation is the preferred orientation of the ITO films deposited using evaporation methods ${ }^{4}$ while the $(400)$ orientation is dominant for the films grown using sputtering method. ${ }^{6}$ The obtained XRD results are thus consistent with these previous works. The appearance of the (222) diffraction peaks in the XRD curves of the annealed ITO films unambiguously indicates the formation of $\mathrm{In}_{2} \mathrm{O}_{3}$ and $\mathrm{In}_{2} \mathrm{O}_{x} \mathrm{Sn}_{y}$ polycrystalline domains during annealing. The broad background seems to be annealing-gas dependent. The film annealed in forming gas is almost the same background as that of the as-deposited film. The broad background in the XRD curves of the films annealed in both $\mathrm{N}_{2}$ and air becomes relatively weaker although the (222) diffraction peak of the film annealed in $\mathrm{N}_{2}$ is likely more clear. Strikingly, the broad background in the XRD curve (top curve) of the film annealed in Ar disappears. However, the (222) diffraction peak of the film is also rather weak. The reason causing this phenomenon is not clear and requires further study.

Figure 4 shows the XPS O $1 s$ data of the films asdeposited and annealed in different annealing gases. These data indicate significant differences in both the chemical states and the oxygen concentration for the films annealed in different annealing gases. Compared with the as-deposited film, the relative intensity of the $\mathrm{O}_{\sim 532}$ peak of the airannealed film increases. The opposite behavior is, however, observed for the films annealed in other annealing gases, i.e., the relative intensity of the $\mathrm{O}_{\sim 532}$ peak decreases with respect to the as-deposited film. The decrease of the $\mathrm{O}_{\sim 532}$ peak intensity is the largest for the film annealed in forming gas while the decrease is the smallest for the film annealed in Ar. Actually, each O $1 s$ curve in Fig. 3 can be decomposed into three different components at binding energies of $530.3 \pm 0.1 \mathrm{eV}, \quad 530.8 \pm 0.3 \mathrm{eV}$, and $532.2 \pm 0.7 \mathrm{eV}$ using combined Gaussian and Lorentzian functions. In the following discussion, $\mathrm{O}_{530.3}, \mathrm{O}_{530.8}$, and $\mathrm{O}_{532.2}$ are employed to designate these three components. The open squares in Fig. 4 are the best fitting curves to the experimental spectra (solid lines). It can be observed that the relative intensity ratios between these three decomposed peaks vary with the annealing environment. The chemical state of the oxygen is thus different for the ITO films annealed under different ambient
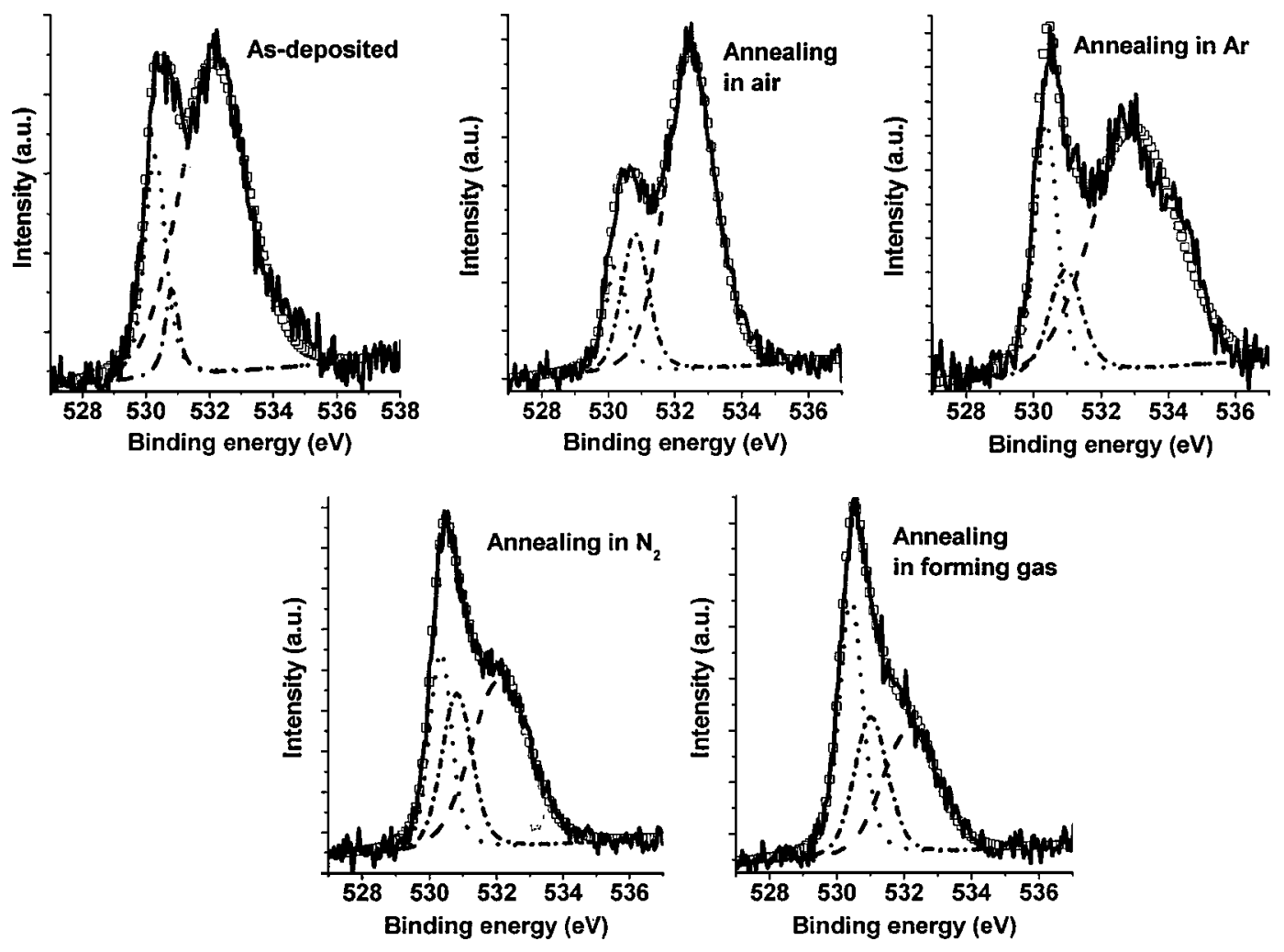

FIG. 4. O $1 s$ XPS curves of the ITO films as-deposited and annealed in different gas environments. 
gases. In a previous study it was observed that the $\mathrm{O}_{532.2}$ peak and the $C$ peak were completely removed after sputtering the samples ${ }^{10}$ for 4 min to remove the top layer of ITO film, from which it was inferred that the $\mathrm{O}_{532.2}$ peak originates from the top surface oxygen contamination ${ }^{5}$ or absorbed water. It is recognized further that the $\mathrm{O}_{530.3}$ and the $\mathrm{O}_{530.8}$ lines arise from the $\mathrm{In}_{2} \mathrm{O}_{3}$ region and the oxygen deficient region $\mathrm{In}_{2} \mathrm{O}_{3-x}$ of ITO film, ${ }^{9}$ respectively.

Now we consider the integrated intensity ratio of $\left(\mathrm{O}_{530.3}+\mathrm{O}_{530.8}+\mathrm{O}_{532.2}\right) /(\mathrm{In}+\mathrm{Sn})$ and the ratio $\mathrm{O}_{530.3} / \mathrm{O}_{530.8}$ in detail. These ratios, which are given in Table I, vary according to the different gas environments. Oxygen incorporation and decomposition reactions in the films during annealing process should be responsible for the observed composition variation. First, the oxygen incorporation reaction is discussed. It has been reported that the film asdeposited by an e-beam evaporation generally contains metal phase indium as the $\mathrm{In}_{2} \mathrm{O}_{3}$ source is used. ${ }^{9}$ When the film was annealed, residual oxygen atoms inside the film, on the top surface, and at the interface between the film and substrate may interact with In atoms to form the $\operatorname{In}_{2} \mathrm{O}_{3-x}$ and the $\mathrm{In}_{2} \mathrm{O}_{3}$ phases. Thus oxygen is incorporated mainly through the direct oxidation of the metal phase In. ${ }^{9}$ However, oxygen incorporation can possibly also occur through absorption into the oxygen deficient phase $\mathrm{In}_{2} \mathrm{O}_{3-x}$ to form $\mathrm{In}_{2} \mathrm{O}_{3}{ }^{9}$. These oxygen incorporation reactions are reflected by the observed variation of $\mathrm{O} / \mathrm{In}+\mathrm{Sn}$ intensity ratio. From the XPS data listed in Table I, the ratio increases from 2.1 for the asdeposited film to 2.3 and 2.4 as the films annealed at $200{ }^{\circ} \mathrm{C}$ in air and Ar, respectively. It is most likely that the oxygen incorporation and oxygen diffusion are responsible for the observed transmittance improvement for the annealed samples. This is consistent with the observation of Mori et $a l .{ }^{12}$ In the Ar annealing case, the increase of $\mathrm{O} / \mathrm{In}+\mathrm{Sn}$ ratio suggests that oxygen diffuses out from the bulk to the surface. That oxygen diffusion are beneficial to form an $\mathrm{In}_{2} \mathrm{O}_{3}$ phase during the annealing process in inert gas and oxygen environment. The $\mathrm{O}_{530.3} / \mathrm{O}_{530.8}$ ratio data tend to support this picture. From the values listed in Table I, it can be found that the larger the $\mathrm{O}_{530.3} / \mathrm{O}_{530.8}$ ratio, the higher the transmittance of the ITO films, except for two cases, the as-deposited films and films annealed in air. The former case can be readily understood since the as-deposited film consists of tiny nanocrystal structures and/or amorphous phase from the XRD data shown in Fig. 3. It is well known that the optical and electrical properties of deposited films can be significantly improved through annealing films at adequate temperatures due to crystallization. ${ }^{16}$ The much poorer transmittance, lower mobility, and larger resistance of the as-deposited film are related to the nanosized and/or amorphous structures ${ }^{4}$ of the film having more grain boundaries that increase grainboundary scattering probability and thus reduce the conductivity as well as the transmittance of the film. In the latter case, $\mathrm{O}_{530.3} / \mathrm{O}_{530.8}$ ratio for films annealed in air is only 0.6 , which is significantly smaller than the ratios observed for the films annealed in $\mathrm{N}_{2}$, Ar, and forming gas. This is probably because the $\mathrm{In}_{2} \mathrm{O}_{3-x}$ phase increases faster than the $\mathrm{In}_{2} \mathrm{O}_{3}$ phase during the In oxidation process in air annealing. From Fig. 5 it can be found that the In $3 d$ peak of the film annealed

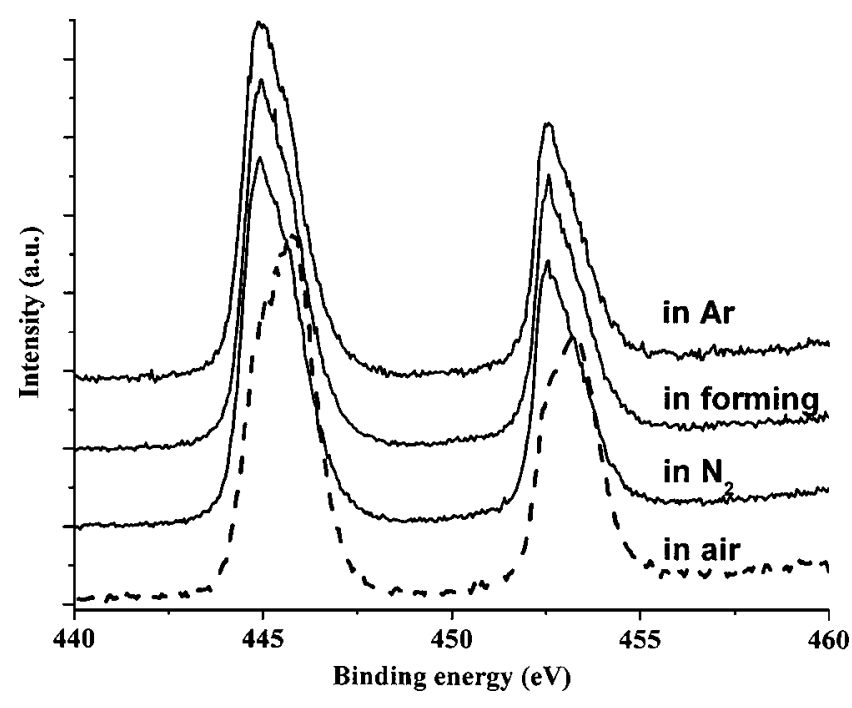

FIG. 5. In $3 d$ XPS curves of the ITO films annealed in different gas environments.

in air clearly shifts to the higher binding-energy side compared with the In $3 d$ peak of the film annealed in other gas environments. When one metal combines with oxygen to become a compound, its inner-shell electron binding increases slightly. The increased binding energy of the In $3 d$ peak for the film annealed in air indicates that the In metal phase is converting into the indium-oxide phase by reacting with oxygen in air. This supports the picture of metallic In removal as the main reason for the observation of the best transmittance of the film annealed in air.

The oxygen decomposition reaction which is reflected by the $\mathrm{O} / \mathrm{In}+\mathrm{Sn}$ ratio variations of the films annealed in $\mathrm{N}_{2}$ and forming gas with respect to the as-deposited film will be discussed in the following. The oxygen loss of the film is proved by a decrease in the ratio from 2.1 for the asdeposited film to 1.6 and 1.5 for the films annealed in $\mathrm{N}_{2}$ and forming gas, respectively. This means that the $\mathrm{N}_{2}$ gas and forming gas are likely to react with the top oxygen layer of the films to, respectively, produce $\mathrm{NO}_{x}$ and $\mathrm{H}_{2} \mathrm{O}$. These reaction products are then carried away by the flowing annealing gases during the annealing process. That reaction has probably two effects on the ITO film microstructure and chemical composition. One possibility is the reduction of the concentration of oxygen inside and at the surface. This is because oxygen diffuses continually out from bulk to surface and $\mathrm{H}_{2} \mathrm{O}$ or $\mathrm{NO}_{x}$ products are carried away during the annealing process mentioned above. As a result, the rate of In-O bonds formation will decrease. Another possibility is oxygen decomposition. Both of these slow down the formation of $\mathrm{In}_{2} \mathrm{O}_{3}$ phase compared with that of the film annealed in inert and oxygen gas atmosphere. It is thus believed that the oxygen decomposition reaction and the slow formation of the In-O causes the observed differences in transmittance and surface morphology of the films annealed in $\mathrm{N}_{2}$ and forming gas. In spite of the existence of the oxygen decomposition reaction for the films annealed in $\mathrm{N}_{2}$ and forming gas, the occurrence of the other competing processes including the increase of crystallinity still give rise to improvements in the optical and electrical properties of the ITO films annealed in these gases. 


\section{CONCLUSIONS}

The ITO thin films deposited on glass using e-beam evaporation have been annealed in different ambient gases. The XPS results reveal that the oxygen incorporation and decomposition reactions take place during postdeposition annealing. Both competing reactions depend on the annealing gases. These reactions substantially influence the carrier concentration and chemical states in the annealed ITO films and thus, in turn, strongly change the optical transmission and electrical properties of the films. In particular, our results indicate that oxygen incorporation into the ITO film is beneficial for transmittance in two aspects, the first being the incorporation of oxygen into the $\operatorname{In}_{2} \mathrm{O}_{3-x}$ phase to form $\operatorname{In}_{2} \mathrm{O}_{3}$ and the second the removal of metallic In to form both indium oxide phases.

\section{ACKNOWLEDGMENTS}

The work described in this paper is partially supported by the grants from the Research Grant Council of the Hong Kong Special Administrative Region, China (under Project Nos. HKU7021/04P, HKU7103/02P, HKU7004/03P, and HKU1/00C).
${ }^{1}$ J. K. Sheu, Y. K. Su, G. C. Chi, M. J. Jou, and C. M. Chang, Appl. Phys. Lett. 72, 3317 (1998)

${ }^{2}$ H. Y. Chen, C. F. Qiu, M. Wong, and H. S. Kwok, IEEE Electron Device Lett. 24, 315 (2003).

${ }^{3}$ H. Kobayashi, T. Ishida, K. Nakamura, Y. Nakato, and H. Tsubomura, J. Appl. Phys. 72, 5288 (1992).

${ }^{4}$ A. Salehi, Thin Solid Films 324, 214 (1998).

${ }^{5}$ L. Chkoda, C. Heske, M. Sokolowski, E. Umbach, F. Steber, J. Staudigel, M. Stö $\beta$ el, and J. Simmereer, Synth. Met. 111, 315 (2000)

${ }^{6}$ P. Thilakan and J. Kumar, Vacuum 48, 463 (1997).

${ }^{7}$ I. Baía, M. Quintela, L. Mendes, P. Nunes, and R. Martins, Thin Solid Films 337, 171 (1999).

${ }^{8}$ J. L. Huang, Y. T. Jah, B. S. Yau, C. Y. Chen, and H. H. Lu, Thin Solid Films 370, 33 (2000).

${ }^{9}$ J. C. C. Fan and J. B. Goodenough, J. Appl. Phys. 48, 3524 (1977).

${ }^{10}$ R. X. Wang, C. D. Beling, A. B. Djurišić, S. Li, and S. Fung, Semicond. Sci. Technol. 19, 695 (2004).

${ }^{11}$ T. Ishida, H. Kobayashi, and Y. Nakato, J. Appl. Phys. 73, 4344 (1993).

${ }^{12}$ N. Mori, S. Ooki, N. Masubuchi, A. Tanaka, M. Kogoma, and T. Ito, Thin Solid Films 411, 6 (2002).

${ }^{13}$ D. V. Morgan, A. Salehi, Y. H. Aliyu, and R. W. Bunce, Renewable Energy 7, 205 (1996).

${ }^{14}$ F. D. Aurent, S. D. Goodman, F. K. Koschnick, J. M. Spaeth, B. Beaumont, and P. Gibart, Appl. Phys. Lett. 74, 2173 (1999).

${ }^{15}$ L. Meng, A. Macarico, and R. Martins, Vacuum 46, 673 (1995).

${ }^{16}$ P. Thilakan and J. Kumar, Mater. Sci. Eng., B 55, 195 (1998). 